pneumonia, and in cases of intolerance, dapsone or pentamidine can be used as alternative treatment options.

Methods Retrospective review of patient notes and electronic patient records, for those patients who have completed treatment for Acute Lymphoblastic Leukaemia on the UKALL2003 protocol at the Royal Marsden Hospital.

Results 164 patients were commenced on co-trimoxazole, and one was commenced on dapsone. $22(13.4 \%)$ of patients did not tolerate co-trimoxazole, with 20 cases of intolerance secondary to cytopenia.

Of the 22 patients who discontinued co-trimoxazole, 20 patients $(87 \%)$ have documentation of G6PD status being investigated, all of whom were negative for G6PD deficiency.

The first 5 patients to require 2 nd line therapy were commenced on pentamidine, with 'cytopenia' cited as the cause of co-trimoxazole intolerance for all of these patients. One patient was subsequently changed onto dapsone.

17 patients were commenced on dapsone as 2 nd line therapy for PCP prophylaxis, with 'cytopenia' cited as the cause of co-trimoxazole intolerance in 15 patients.

5 of the 17 patients who were commenced on dapsone as 2 nd line treatment, did not tolerate dapsone. Of these five patients, one had persistent neutropenia, two developed methaemaglobinaemia, and two developed dapsone syndrome.

Only one of 165 patients was suspected of developing pneumocystis pneumonia, and retrospectively this patient was found to be non-compliant with co-trimoxazole.

Conclusions Current PCP prophylaxis treatments are effective, and for the majority of patients, co-trimoxazole is well tolerated.

\section{LATE EFFECTS IN SURVIVORS OF INFANT LEUKEMIA IN SINGLE CENTER}

doi:10.1136/archdischild-2012-302724.0767

'S Ansari, 'A Shir Ali, ${ }^{2}$ S Ziaie, 'P Vossough. 'Tehran University of Medical Sciences; ${ }^{2}$ Shahid Beheshti University of Medical Sciences, Tehran, Iran

Background Acute lymphoblastic leukemia (ALL) is the most common childhood malignancy, accounting for $30 \%$ of all cancers occurring in childhood .long term sequel of treatment are now being reported. Children who survive acute lymphoblastic leukemia are at risk for leukemia-related or treatment-related complications.

Methods In this study we evaluated 66 patients with ALL have survived for more than5 years after diagnosis. Long-term squeal of treatment, such as impaired intellectual and psychomotor functioning, neuroendocrine abnormalities, impaired reproductive capacity, cardio toxicity, and second malignant neoplasm's, are being reported.

Results of the 66 patients, 43 cases were male and 23 female. Mean age was $14.59 \pm 4.36$ (range 10-25 years). 42 patients received chemotherapy alone, 24 patients who received chemotherapy and CNS radiation therapy. Short height $33 / 3 \%$,over weight $50 \%$, low bone density $53 \%$, learning disabilities $6 / 1 \%$, hyperthyroidism $1 / 5 \%$, sexual development (pubertal delay) $7 / 6 \%$, over weight are more common in children who get chemotherapy without radiotherapy. $31 / 8 \%$ of patients don't have late effects. $30 / 3$ had at least one late complication.

Conclusion These results indicate that late sequelae are common in long term survivors of infant leukemia and are often related to CRT the most common problem are short stature and over weight.

\section{EVALUATION OF SERUM TRANSFERRIN RECEPTOR IN PATIENTS AFFECTED BY IRON-DEFICIENCY ANEMIA AND COMPARISON WITH CONTROL GROUP}

doi:10.1136/archdischild-2012-302724.0768
'S Shams, ${ }^{2} \mathrm{SO}$ Mohammadian, 'M Monajemzadeh, ${ }^{2} \mathrm{H}$ Irani, ${ }^{2} \mathrm{~L}$ Shafaghat, 'MT Haghi Ashtiani. 'Pathology Department; ' $C$ enter of Excellence for Pediatrics, Children's Medical Center, Tehran University of Medical Sciences, Tehran, Iran

Background and Aims Iron deficiency anemia is one of the most common nutritional deficiencies and public health around the world. The growing Children are one of groups that are at high risk for this problem. Iron deficiency anemia can cause mental, motor or behavior problems. So early diagnosis and treatment can prevent great side effects. One of the diagnosis means is measuring of soluble tranferrin receptor level (sTfR) in serum because level of this receptor increase in result of iron depletion in iron deficiency but has no change in other.

Methods and materials: This was a case control study included 64 children with iron deficiency anemia (IDA) and healthy subjects. The study conducted, in 2008-2010 in Children Medical Center. Blood samples collected from every patient in case and control groups and $\mathrm{Hb}, \mathrm{MCV}, \mathrm{Fe}$, Ferritin, TIBC and sTfR measured and compared in the groups.

Results Compared to the control group, serum sTfR mean level was significantly higher in children with IDA than control group $(1.87 \mathrm{vs} .1 .06 \mu \mathrm{g} / \mathrm{ml}, \mathrm{P}$ value $=0.002) . \mathrm{sTfR}$ showed negative correlations with $\mathrm{Hb}(\mathrm{r}=-0.629, \mathrm{p}<0.001), \mathrm{MCV}(\mathrm{r}=-0.649, \mathrm{p}<0.001)$ and serum Ferritin $(r=-0.224, p=0.053)$, although it was not significant for Ferritin. There was no significant differences between cases and controls in sTfR mean level with regard to gender and age categories. $(\mathrm{P}>0.05)$.

Conclusions This study shows sTfR level can be an appropriate biomarker for diagnosis of IDA, particularly in patient with IDA coexisting with inflammation.

\section{EXCESSIVE MENSTRUAL BLEEDING IS A RISK FACTOR OF ANEMIA IN ADOLESCENT SCHOOLGIRLS}

doi:10.1136/archdischild-2012-302724.0769

I Tarasova, V Chernov. Child Hematology and Oncology, Federal Research Center of Pediatric Hematology, Oncology, and Immunology named after Dmitry Rogachev, Moscow, Russia

Background and Aims Adolescents form a group at risk of iron deficiency development. An additional factor leading to depletion of iron stores in adolescent girls is blood loss during menstrual bleeding.

Methods A cross-sectional nonrandomized descriptive study was carried out in order to evaluate the incidence of dysfunctional uterine bleeding (DUB) in adolescent girls and detect the relationship between menstrual blood loss and anemia, in order to develop measures to improve the health status of adolescents. The study was carried out within the routine health examination of schoolchildren in Vologda. A total of 1340 girls aged 10-18 years (mean age $13.81 \pm 0.04$ years) were selected at random. The menstrual function was studied in 883 girls aged $11-18$ years (mean age $14.5 \pm 0.04$ years) by the data of questionnaires and results of gynecological examinations.

Results The prevalence of anemia ( $\mathrm{Hb}$ less than $120 \mathrm{~g} / \mathrm{l}$ ) in all examined girls of Vologda was $10.7 \%(n=143)$, in adolescent girls with menses $-10.5 \%(n=93)$. The age of menarche varied from 10 to 16 years (mean age $12.57 \pm 0.03$ years). DUB were detected in 54 (6.2\%) girls. Anemia was detected in 11 (20.4\%) girls with DUB. Of the menstrual characteristics excessively heavy menstrual bleeding was the only risk factor for anemia $(\mathrm{OR}=0.43,95 \% \mathrm{CI}=0.22-0.87$, $p=0.0348$ ).

Conclusions Menstrual iron loss is the main risk factor for iron deficiency development, and hence, in order to detect the causes of anemia in females of reproductive age the complete anamnesis on the pattern of menstruation should be collected. 